Preprints of the

Max Planck Institute for

Research on Collective Goods

Bonn 2006/7

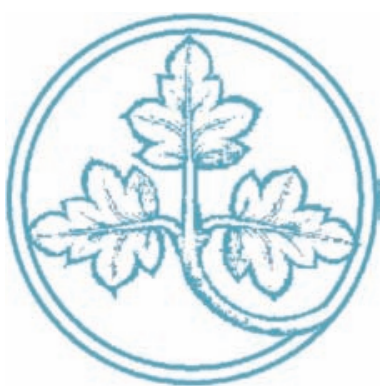

Zur Interaktion von Recht und sozialen Normen bei der dezentralen Bereitstellung von Gemeinschaftsgütern

Stefan Magen

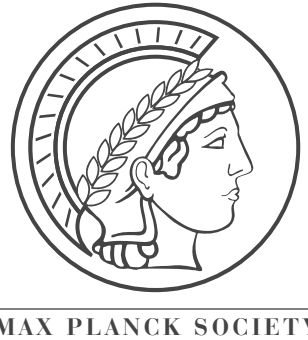




\title{
Zur Interaktion von Recht und sozialen Normen bei der dezentralen Bereitstellung von Gemeinschaftsgütern
}

\author{
Stefan Magen
}

March 2006 


\title{
Zur Interaktion von Recht und sozialen Normen bei der dezentralen Bereitstellung von Gemeinschaftsgütern
}

\author{
von Stefan Magen ${ }^{1}$
}

\section{Einleitung}

Sowohl Recht also auch soziale Normen können zur Lösung sozialer Probleme beitragen, aber sie tun dies in unterschiedlichen Formen und sie stehen dabei in undeutlicher Weise unter gegenseitigem Einfluss. ${ }^{2}$ Vergleichsweise klar sind ihre strukturellen Unterschiede, wie sie mit der Kennzeichnung als formale und informale Institutionen hervorgehoben werden: Rechtsnormen werden (im Regelfall) von Organisationen in förmlichen Verfahren geschaffen und autoritativ durchgesetzt. Soziale Normen entspringen diffusen, ungeregelten und nicht auf einzelne Akteure zurechenbaren gesellschaftlichen Prozessen. Aber auch hinsichtlich des Schemas von Zentralität und Dezentralität scheint zwar keine trennscharfe Zuordnung, aber doch eine Zuordnung der Tendenz nach immer noch sinnvoll. In erheblichem Ausmaß wird das Recht nach wie vor von zentralstaatlichen oder föderalen Instanzen zentral gesetzt (im Fall supranationaler Institutionen vielleicht sogar „hyperzentral“) und soziale Normen meist mehr oder weniger lokal situiert. Und auch wenn die Zentralität des Rechts in Bewegung geraten sein sollte, sich für manche gar in einen Pluralismus der Rechtsordnungen aufzulösen scheint, bleibt für viele soziale Normen doch die Verankerung in vergleichsweise dezentralen sozialen Konstellationen prägend.

Das ist der Ausgangspunkt dieser Analyse, die sich dafür interessiert, welche Bedeutung die Existenz bestimmter sozialer Normen für die Problemlösefähigkeit des Rechts hat. ${ }^{3}$ Um deren

1 Für Kritik und hilfreiche Anmerkungen danke ich Christoph Engel, Martin Hellwig und Andreas Nicklisch.

2 Das Verhältnis von Recht und sozialen Normen wurde unter wechselnden Überschriften (Volksgeist, Sitte, Sozialmoral, Rechtspluralismus, informale Institutionen, soziale Normen, etc.) von diversen Disziplinen behandelt, etwa der historischen Rechtsschule (Savigny, 1814), der (Rechts-)Soziologie (Summner, 1940; Tamanaha, 2001), der (Rechts-)Anthropologie, der Institutionenökonomie (Mantzavinos 2001) und der ökonomischen Analyse des Rechts (Ellickson, 1991).

3 Weil die Fähigkeit des Rechts, soziale Probleme zu lösen, weiter reichen kann, als die Fähigkeit zentraler Akteure, gesellschaftliche Prozesse zentral zu „steuern“, vermeiden wir hier den Begriff der Steuerungsfähigkeit. 
Interaktion zu verstehen, ist es aber nicht befriedigend, soziale Normen als substanzhafte Entitäten zu behandeln, die als soziale Einbettung allem Entscheiden vorausliegen (vgl. Beckert, 2003). Das soll nicht in Abrede stellen, dass sich soziale Normen und andere aus Interaktionsprozessen emergierende Phänomene gegenüber den Individuen verselbständigen. Aber wesentliche Aspekte des Zusammenspiels von Recht und sozialen Normen lassen sich nicht adäquat beschreiben, ohne auch die Mechanismen der Prozesse in den Blick zu nehmen, in denen informale Institutionen aus der Interaktionen der Individuen hervorgehen (vgl. Opp, 2001). Im Folgenden wird deshalb das Verhältnis von Recht und sozialen Normen aus der Warte individuellen Entscheidens thematisiert. Die Analyse beginnt mit rationaltheoretischen Ansätzen, die dann im Sinne der Verhaltensökonomie durch empirische Forschungen aus Laborexperimenten und Feldstudien modifiziert werden (vgl. Ostrom, 2003). Betrachtet werden allerdings nur soziale Normen (informale Institutionen, deren Verletzung informale Sanktionen nach sich zieht), die der Bewältigung öffentlicher (mehrere Personen treffender) Externalitäten dienen (vgl. Coleman, 1990; Arce/Sandler, 2005). Diese Probleme haben häufig (aber nicht notwendig) die Struktur eines n-Personen Gefangen-Dilemmas (Coleman, 1990; Hechter, 1990; Heckathorn, 1996). Man spricht insoweit von Problemen kollektiven Handels (Olson 1998; R. Hardin, 1982) oder von sozialen Dilemmata (Beckenkamp, 2002; Dawes 1980). Die Anwendungsbeispiele sind wiederum nur einer Unterklasse von Problemen entnommen, nämlich den Gemeingutsproblemen (G. Hardin, 1968; Ostrom et al. 2002). Es wird also versucht, von Rationaltheorie, Laborexperimenten und Feldstudien darüber zu lernen, wie zentrale und lokale Institutionen bei der Lösung von Gemeingutsproblemen zusammenwirken. Es steht zu hoffen, dass diese Einsichten auch für andere Probleme kollektiven Handelns wie auch generell für das Verständnis der Interaktion von Recht und sozialen Normen hilfreich sind.

\section{Das Gemeingutsproblem}

Fischgründe, offenes Weideland, knappe Wasserressourcen oder eine saubere Umwelt sind Beispiele für Gemeingüter (oder auch Allmenden oder gemeinschaftlich genutzte Ressourcen common pool resources - genannt). Vor allem aufgrund zweier Eigenschaften sind solche Güter nach gängiger ökonomischer Analyse der Gefahr der Übernutzung ausgesetzt: aufgrund der Nichtausschließbarkeit des Gebrauchs und aufgrund der Rivalität des Gebrauchs (Fritsch/Wein/ Ewers, 2005, 101). ${ }^{4}$ Nichtausschließbarkeit meint, dass weitere Nutzer aufgrund der natürlichen Beschaffenheit des Guts nicht oder nur unter prohibitiven Kosten von dessen Gebrauch ausgeschlossen werden können. Das Übernutzungsproblem stellt sich aber gleichermaßen, wenn der fehlende Ausschluss nur eine Folge der die Nutzung regelnden Institutionen ist, und nicht auf den natürlichen Eigenschaften des Gutes beruht. Rivalität bedeutet, dass der Gebrauch einer Einheit der Ressource deren Gebrauch durch einen anderen ausschließt. So stehen die von einem Fischer gefangenen Fische den anderen nicht mehr zur Verfügung. Der Gebrauch von Nutzeneinheiten kann aber auch - zumindest ab einem bestimmten Ausmaß der Nutzung - den Ertrag

$4 \quad$ Umweltgüter können beispielsweise insofern Gemeingüter sein, als sie zur Entsorgung von Abfall oder zur

Aufnahme von Schadstoffen genutzt werden (Dietz et al., 2002: 19). 
der Ressource insgesamt beeinträchtigen und damit zusätzliche negative Externalitäten verursachen (Ostrom et al. 1994: 10 f.). Kann zum Beispiel jeder auf eine Ressource, die sich nicht beliebig regeneriert, frei zugreifen, wird sie schnell übernutzt: die Fischgründe überfischt, das Weideland überweidet, die Wasserquelle erschöpft, und die Umwelt verdreckt. Dann endet man leicht in einer Situation, in der alle schlechter dastehen, als wenn sie mit der Ressource schonender umgegangen wären. Es läge dann eigentlich im allgemeinen Interesse, wenn jeder sein Verhalten so einrichten würde, dass die Ressource nicht über das sinnvolle Maß hinaus genutzt wird. Solche Zurückhaltung liegt aber nicht auch im Interesse des isolierten Einzelnen, wenn dieser seinen individuellen Ertrag aus der Ressource durch stärkere Nutzung noch weiter steigern könnte. In diesem Auseinandertreten von individueller und sozialer Rationalität liegt das Gemeingutsproblem (Beckenkamp, 2002).

Institutionen, die dieses Problem lösen sollen, kommt entsprechend die Aufgabe zu, Externalitäten durch Übernutzung zu verhindern und die Einzelnen zum Verhalten im gemeinsamen Interesse zu bewegen. Dies kann im Prinzip auf zwei Weisen geschehen (vgl. Kollock 1998). Erstens können die Rahmenbedingungen so verändert werden, dass der Einzelne kein materielles Interesse mehr hat, die Ressource über das sozial optimale Maß hinaus zu nutzen. Das wird vor allem durch Sanktionen erreicht, die die sozial unerwünschte Nutzung mit einem materiellen Nachteil belegen und dadurch den individuellen Anreiz zur Übernutzung aufheben. Diese Aufgabe wird häufig vom Recht übernommen, indem es sanktionsbewehrte Verbote ausspricht. Eine Korrektur der materiellen Anreize kann aber auch durch soziale Normen erreicht werden, wenn deren Verletzung mit materiellen Sanktionen geahndet wird. Zweitens können Gemeingutsprobleme aber auch bewältigt werden, wenn die Akteure in einer Weise motiviert sind, die sie dazu veranlasst, dem Anreiz zur Übernutzung nicht nachzugeben. Über diesen motivationalen Pfad wirken beispielsweise soziale Normen, die internalisiert sind (Cooter, 1998) oder durch Kundgabe von Missbilligung durchgesetzt werden (Rege, 2004). ${ }^{5}$ Die größte Aufmerksamkeit haben in jüngeren empirischen Forschungen aber soziale Normen auf der Grundlage von Gegenseitigkeit erfahren. Gegenseitigkeitsnormen beruhen nun sowohl auf nicht-eigennützigen Motiven als auch auf einer Korrektur der materiellen Anreize, wobei beide Elemente in einer bestimmten Weise zusammenwirken, wie später ausgeführt werden wird.

\section{Rationaltheoretische Ansätze}

Die prägnanteste Analyse der von Gemeingütern aufgeworfenen Anreizprobleme erlauben Theorien, die mit der Annahme rationalen egoistischen Verhaltens arbeiten (vgl. Kirchgässner, 2000). Solche Ansätze modellieren das Gemeingutsproblem häufig mit den Konzepten der Spieltheorie als ein Gefangenen-Dilemma (Fritsch/Wein/Ewers, 2005, 103; Weimann, 1995, 60; differenzierter Heckathorn, 1996).

5 Auch diese beiden Motive lassen sich als Sanktionen modellieren. Allerdings ändern psychische „Kosten“, wie sie aus öffentlichem Tadel oder der Übertretung internalisierter Normen resultieren können, die materiellen Anreize nicht, sondern begründen zusätzliche, immaterielle Anreize. 


\section{a) Gemeingüter als Gefangenen-Dilemmata}

Angenommen, zwei Landwirte teilen sich eine Wasserquelle, die sich ab einer bestimmten Nutzung nicht mehr ausreichend regeneriert. Jeweils stehen sie vor der Entscheidung, ob sie ihr Feld intensiv oder sparsam bewässern sollen. Der Gesamtertrag aus den Feldern ist am höchsten, wenn beide die Quelle sparsam nutzen. Das wäre die - sozial erstrebenswerte -kooperative Lösung des Problems. Allerdings kann einer der Landwirte einen noch höheren Ertrag erwirtschaften, wenn er die Quelle einseitig intensiv nutzt, sich also opportunistisch verhält oder „defektiert“. Die intensive Nutzung durch den einen hat allerdings negative Externalitäten für den anderen, sparsamen Landwirt, dessen Ertrag auf ein Minimum fällt. Er kann allerdings seine Lage etwas verbessern, wenn er ebenfalls zu intensiver Nutzung übergeht. Dadurch steigt sein relativer Anteil, aber der Gesamtertrag sinkt noch weiter. Beide stehen nun schlechter da, als sie bei beiderseitiger Sparsamkeit stehen würden. Das ist die unkooperative Lösung, in die die Landwirte durch Gier nach dem Defektionsgewinn und die Furcht vor Ausbeutung getrieben werden (vgl. Macy/Flache 2002). Sie scheint unter der Annahme eigennütziger Akteure unvermeidlich, weil eine intensive Nutzung auf jedes Verhalten des Gegenübers die ertragreichere Reaktion ist. Durch Garrit Hardins berühmt gewordenen Aufsatz über die „Tragödie der Gemeingüter“ (1968) prägte diese Einschätzung über lange Zeit die Sicht auf Gemeingüter. Ohne staatliche Intervention schien deren Übernutzung unvermeidlich und häufig wurden aus dieser Sorge lokale Ressourcen der Verwaltung durch staatliche Behörden unterstellt. Dabei übersah man aber allzu oft, dass die Nutzung der gemeinschaftlichen Ressourcen auf lokaler Ebene durch informale Institutionen geregelt war. Im Vergleich zu diesen lokalen Institutionen waren die neu eingeführten staatlichen Regime weniger auf die lokalen Verhältnisse abgestimmt, konnten das Nutzungsverhalten nicht in dem gleichen Ausmaß wie die lokalen Gemeinschaften überwachen und sanktionieren und waren häufig auch von Korruption und Misswirtschaft geplagt. Die wahre Tragödie der Gemeingüter setzte dann nicht selten erst mit der staatlichen Regulierung ein (Ostrom, 2005a: 266; Ostrom et al. 1999; Trawick 2002).

Die möglicherweise zu pessimistischen Schlussfolgerungen mancher rationaltheoretischer Ansätze resultieren allerdings zum Teil aus der Annahme, dass die Beteiligten nur einmalig miteinander interagieren oder jedenfalls über die Nutzung jeweils isoliert entscheiden. Vorwiegend ist das Gemeingutsproblem in der Literatur zunächst in der Tat als ein einmaliges GefangenenDilemma modelliert worden (R. Hardin, 1982; vgl. Hackathorn, 1989, 79). Viele - aber keineswegs alle - Gemeingutsprobleme betreffen aber Situationen, in denen die Akteure regelmäßig miteinander zu tun haben. So interagieren Landwirte, die gemeinsam eine Wasserquelle nutzen, für gewöhnlich über einen längeren Zeitraum miteinander. Spieltheoretisch gesprochen spielen sie das Gefangenen-Dilemma dann als ein unbestimmt oft wiederholtes Spiel. Dadurch verschwinden die ungünstigen Anreize nicht, aber es eröffnen sich verschiedene Möglichkeiten, das Dilemma zu lösen (Baird et al. 1998: 165 ff.; Heckathorn, 1989). Angenommen, die beiden Landwirte müssen in jeder Bewässerungsperiode erneut über die Intensität der Bewässerung entscheiden. Wer eine bestehende Kooperation abbricht und einseitig zur intensiven Bewässerung übergeht, kann dann zwar seinen Ertrag in der nächsten Periode steigern, riskiert aber, dass 
der andere dem folgt und er auf unbestimmte Zeit mit den niedrigeren Erträgen der unkooperativen Lösung auskommen muss. Auch für rationale Egoisten ist es dann eine gewinnbringende Strategie, die Zurückhaltung des Gegenübers mit eigener Zurückhaltung zu beantworten, intensive Nutzung aber mit intensiver Nutzung zu strafen (Kreps, 1990, 97 ff.). Dieser Logik folgen Strategien wie das bekannte „Tit-for-Tat“ (Axelrod 1984).

Auch damit hat man freilich noch keine befriedigende Vorstellung von Gemeingutsproblemen. Der Grund ist, dass die kooperative Lösung nur unter unrealistischen Annahmen auf Situationen übertragen werden kann, in denen ein Gemeingut von vielen Personen genutzt wird (Olson, 1998; Beckert, 1997: 45; Weimann, 1995: 69). Wenn nicht zwei, sondern zehn Landwirte eine Quelle nutzen, und das opportunistische Verhalten eines einzelnen Landwirts nur dadurch sanktioniert werden kann, dass auch die anderen sich unkooperativ verhalten, gerät Kooperation zu einer fragilen Angelegenheit. Eine solche Sanktion träfe nämlich auch die anderen, kooperativen Landwirte, die ihrerseits wiederum mit Sanktionen reagieren könnten. Ohne zusätzliche Mechanismen würde Kooperation deshalb rasch zusammenbrechen, sobald einer der Beteiligten zum Trittbrettfahren übergeht (R. Hardin, 2000, 1826).

Dieses Problem kann durch selektive Sanktionen vermieden werden, die nur den unkooperativen Akteur treffen, etwa indem einem unkooperativen Landwirt eine sonst übliche Hilfe bei der Ernte verweigert wird. Damit zeichnet sich nun eine adäquatere Beschreibung der Möglichkeiten und Grenzen rationaler Kooperation und damit der Anreizprobleme von Gemeingütern ab: Für den einzelnen ist die Schonung der Ressource nur rational, wenn mit hinreichender Wahrscheinlichkeit zu erwarten ist, dass übermäßige Nutzung gezielt sanktioniert wird. Das setzt zunächst voraus, dass Normbrüche mit hinreichender Wahrscheinlichkeit entdeckt werden (Hechter, 1990: 16). Überwachung und Sanktionierung sind aber häufig mit Kosten verbunden, weil für das Überwachen Zeit und Ressourcen aufgewandt werden müssen und derjenige, der andere sanktioniert, mit Vergeltungsmaßnahmen rechnen muss. Weil alle von den höheren Erträgen der Ressource profitieren, wird mit der Durchsetzung von Kooperation ein öffentliches Gut bereitgestellt, das seinerseits mit dem Opportunismusproblem zu kämpfen hat. Denn jeder hat einen Anreiz, die Normdurchsetzung anderen zu überlassen und von den positiven Wirkungen der Sanktionen auf das Kooperationsniveau als Trittbrettfahrer zu profitieren (Coleman, 1990: 52; Ostrom, 2000: 31 f.). Die kooperative Lösung der Ressourcennutzung wirft damit auf der Ebene der Sanktionen ein neues Dilemma auf, ein soziales Dilemma zweiter Ordnung (Heckathorn, 1989: 78 ff.). Die Möglichkeit und die Kosten von Überwachung und Sanktionierung sind damit zentrale Probleme, mit denen Gemeingüter nach dieser Analyse zu kämpfen haben.

\section{b) Soziale Normen als kooperative Gleichgewichte}

Für viele rationaltheoretische Ansätze kann die kooperative Lösung eines Dilemmas nur darin bestehen, dass die Beteiligten Verhaltensweisen finden, in denen das Verhalten jedes einzelnen jeweils eine optimale - nutzenmaximierende - Antwort auf das Verhalten der anderen ist. Die Strategien der Beteiligten befinden sich dann im Gleichgewicht (sog. Nash-Gleichgewicht, vgl. 
Kreps 1990: 28). Unbestimmt oft wiederholte Interaktionen weisen eine Vielzahl derartiger Gleichgewichte auf (sog. Folk-Theorem, vgl. Rasmusen 1994: 124). Auch soziale Normen lassen sich für rationaltheoretische Ansätze als Gleichgewichte in diesem Sinn beschreiben, und zwar als solche, in denen die Strategien die glaubhafte Drohung beinhalten, Normabweichungen zu sanktionieren (Voss, 2001). Soziale Normen resultieren in dieser Sicht aus einer rationalen Koordination von Strategien in einem unbestimmt oft wiederholten Spiel. Ihre Stabilität rührt allein daher, dass es für den einzelnen Beteiligten nachteilig wäre, isoliert von seiner Strategie abzuweichen, nicht aber auf einer irgendwie gearteten Stabilität normativer Erwartungen, wie sie etwa der Begriff der Internalisierung impliziert.

Wenn soziale Normen zur Lösung von Gemeingutsproblemen dieser Logik folgen, hat dies deutliche Implikationen für deren mögliche Reichweite und damit für die Frage, wie lokal bzw. dezentral informale Institutionen in diesem Bereich sein werden. Weil Kooperation nur rational ist, wenn immer wieder die gleichen Beteiligten miteinander interagieren und wenn unkooperatives Verhalten von den anderen Beteiligten mit hinreichender Wahrscheinlichkeit entdeckt und sanktioniert wird, werden informale Ressourcenregime nur in vergleichsweise kleinen Gruppen entstehen können (Ellickson, 1991: „close-knit communities“; Voss, 2001). Unter rationaltheoretischen Prämissen sollte Dezentralität also ein bestimmendes Merkmal sozialer Normen sein.

\section{c) Formale und informale Institutionen}

In seiner Theorie über die Tragödie der Gemeingüter kam es Garrit Hardin nicht zuletzt darauf an, gegen bloße Appelle an individuelle Moral die Notwendigkeit von „Zwang“ zu betonen (G. Hardin, 1968: 1246; ähnlich Lübbe-Wolff, 1999). An dieser Notwendigkeit ändert sich auch dann nichts, wenn man das Gemeingutsproblem nicht mehr als einmaliges, sondern als ein wiederholtes Gefangen-Dilemma begreift, denn auch dann beruht das kooperative Gleichgewicht auf Sanktionen (als Teil einer Gleichgewichtsstrategie, vgl. Voss 2001: 119). Es wird aber einsichtig, dass sich zwischen staatlichem Zwang und unverbindlicher Individualmoral ein weites Feld an potentiellen, informalen und lokalen Lösungen erstreckt, deren Wirkung ebenfalls auf Zwang beruht, allerdings nicht auf staatlichem. Damit stellt sich die Frage, in welchem Verhältnis staatliche und informale, lokale Institutionen zueinander stehen. Hinsichtlich des Anreizproblems läuft dies auf die Frage hinaus, ob die formalen Sanktionen aus dem staatlichen Recht und die informalen Sanktionen aus den lokalen Institutionen gleichläufig oder gegenläufig sind. Denn für eigennützige Akteure ist nur relevant, was die bei unkooperativem Verhalten zu erwartenden Netto-Sanktionen sind. Viele der gescheiterten Versuche in Entwicklungsländern, lokale Ressourcen in staatlicher Verwaltung zu bewirtschaften, lassen sich etwa damit plausibel machen, dass einerseits existierende lokale Institutionen gezielt untergraben und dadurch die Drohung sozialer Sanktionen beseitigt wurden, andererseits aber staatliche Sanktionen wegen mangelnder Verwaltungsressourcen oder durch Korruption zu schwach waren, um ohne lokale Unterstützung eine Übernutzung des Gutes zu verhindern (Ostrom, 2005a: 266). Zwischen formalen und informalen Institutionen muss aber nicht notwendig ein Antagonismus bestehen. Im Gegenteil gibt es 
auch Beispiele dafür, dass Verstöße gegen staatliches Recht auch soziale Sanktionen nach sich ziehen können, also die Durchsetzung der formalen Institutionen durch soziale Normen unterstützt wird (Cooter, 1998). Rechtliche Rauchverbote haben etwa nicht selten zur Folge, dass nun auch die betroffenen Nichtraucher sich beschweren und dadurch zur Durchsetzung des Rechts beitragen (Cooter, 2000).

\section{Präferenzen für Fairness und bedingte Kooperation}

Die bislang behandelten rationaltheoretischen Ansätze basieren auf der Annahme, dass die Akteure nur ihren eigenen Nutzen maximieren. Damit sind Präferenzen für das soziale Wohl ausgeschlossen (sog. Eigennutzenannahme; vgl. Kirchgässner, 2000, 16 f.). Eine solche Beschränkung dessen, was als Inhalt von Präferenzen in Betracht gezogen wird, ist für rationaltheoretische Ansätze üblich, aber nicht zwingend. Insbesondere im Umfeld der Verhaltensökonomik sind Ansätze populär geworden, die das rationaltheoretische Verhaltensmodelle um nicht-eigennützige, „soziale“ Präferenzen ergänzen (Bowles, 2004, 96 f.; für soziologische Ansätze vgl. z.B. Aretz, 2005; Beckert, 2005). Verhaltensökonnomische Ansätze weisen dabei die Besonderheit auf, dass sie bestrebt sind, die Plausibilität von Präferenzannahmen durch Laborexperimente zu stützen (Camerer, 2003). In diesem Bemühen um eine realitätsnähere Modellierung des Verhaltens in Gemeingutsproblemen und anderen sozialen Dilemmata wird insbesondere Präferenzen für Fairness eine zentrale Rolle eingeräumt (Magen, 2006).

\section{a) Positive und negative Reziprozität}

Fairness erscheint dabei in doppelter Gestalt, nämlich als positive und negative Reziprozität, also der Neigung, faires Verhalten mit fairem Verhalten zu erwidern, und dem Bedürfnis, unfaires Verhalten mit Sanktionen zu belegen (Fehr/Gächter, 2000b). In Bezug auf Gemeingüter führt positive Reziprozität zu bedingter Kooperation, nämlich der Bereitschaft, die eigene Nutzung des Gemeinguts auf ein „faires“ Maß zu beschränken, wenn auch die anderen Nutzer des Guts zu solchem kooperativen Verhalten bereit sind. Negative Reziprozität begründet die Bereitschaft, andere zu sanktionieren, wenn diese einen unfairen Gebrauch von dem Gemeingut machen (Fehr/Gächter, 2000a). Anders als die bekannte, rationale Tit-for-Tat-Strategie in wiederholten Spielen entspringt dieses Verhalten aber keinem eigennützigen Kalkül, sondern aus einer (zu einem gewissen Grad) selbständigen Motivation, die neben das Eigennutzstreben tritt. ${ }^{6}$ Allerdings verhält sich in Kollektivgut-Experimenten nur ein Anteil von typischerweise bis zu 60 \% der Menschen in dem geschilderten Sinn reziprok. Diese Teilnehmer kooperieren, wenn die anderen kooperieren, und defektieren, wenn die anderen defektieren. Nur ca. 10 \% der Personen verhalten sich gleichsam als prinzipienfeste Kantianer, kooperieren also unabhängig vom Ver- 
halten der anderen, während sich ca. 30 \% rein eigennützig verhalten (Fischbacher et al., 2001; vgl. auch Kurzban/Houser, 2001). ${ }^{7}$

Wären die reziproken und prinzipienfesten Akteure unter sich, könnte Kooperation auch ohne Sanktionen stabil sein. Sind aber auch eigennützige Akteure beteiligt, bringt deren opportunistisches Verhalten die nur bedingte Kooperation der Reziproken zum Erliegen (Fehr/Fischbacher, 2003). Überwachen und Sanktionieren sind also in verhaltensökonomischer Sicht nicht überflüssig, bekommen aber eine andere Funktion. Ihre Aufgabe ist es nicht mehr, die Anreize in einer Weise zu ändern, dass Kooperation für Jedermann im eigenen Interesse liegt, sondern das Trittbrettfahren der Eigennützigen zu unterbinden, damit die bedingte Kooperation der Reziproken erhalten bleibt. Wesentliche Bedeutung für die Aufrechterhaltung von Kooperation kommt der negativen Reziprozität zu, also der Bereitschaft, unkooperatives Verhalten unter Inkaufnahme eigener Kosten zu sanktionieren. Sie überwindet das Dilemma zweiter Ordnung, in dem jeder versucht, die Kosten für die Durchsetzung von Kooperation den anderen zu überlassen. ${ }^{8}$

\section{b) Soziale Normen als Erwartungsgleichgewichte}

Auch unter der Annahme von Fairnesspräferenzen lassen sich soziale Normen als Gleichgewichte in der Interaktion der Beteiligten analysieren (Kahan, 2005; Traxler, 2006). Im Vergleich zu einer Interaktion eigennütziger Akteure ist Kooperation hier aber bereits unter weniger restriktiven Voraussetzungen zu erwarten. Anders gesagt ist die potentielle Reichweite kooperativer Lösungen mit steigendem Anteil von reziproken Akteuren vermutlich größer (Magen, 2006, 43 ff.; Strahilevitz, 2003). Außerdem hat die Stabilität von Gleichgewichten in Bezug auf diese Akteure einen anderen Anknüpfungspunkt. Während bei eigennützigen Akteuren kooperatives Verhalten primär von der zu erwartenden Sanktion abhängt, ist die Kooperation von reziproken Akteuren eine Reaktion auf die wahrgenommene Kooperationsbereitschaft der Anderen. Unter diesen Akteuren sind kooperative Gleichgewichte dann auch auf der Grundlage geteilter Erwartungen möglich (Kahan, 2005).

\section{c) Aversion vor unfairen Verteilungen}

Freilich können Präferenzen für Fairness die Zahl möglicher Gleichgewichte auch einschränken (Magen, 2006, 51 f.). Der Grund ist, dass faire Akteure Gleichgewichte nicht nur nach ihren isolierten Konsequenzen für den eigenen Nutzen bewerten, sondern auch nach ihren relativen Verteilungseffekten. Nehmen wir an, von den zehn Landwirten in unserem Beispiel haben zwei Landwirte aufgrund der räumlichen Lage ihrer Grundstücke die Möglichkeit, ihre Felder auch aus einer anderen Quelle zu bewässern. Von einer Übernutzung der ersten Quelle sind sie des-

7 Diese drei Verhaltenstendenzen und ihre ungefähre Größenverhältnisse sind in Experimenten recht gut etabliert. Die genauen Prozentzahlen sind dagegen mit erheblicher Vorsicht zu behandeln.

8 Tatsächlich sind sogar unbeteiligte Dritte bereit, Kosten aufzuwenden, um unfaires Verhalten zu bestrafen (Fehr/Fischbacher, 2004). 
halb viel weniger betroffen. Eine solche Außenoption würde ihre Verhandlungsposition bezüglich der Nutzung der ersten Quelle stärken. Unter der Prämisse egoistischen Verhaltens wäre dann zu erwarten, dass diese Landwirte für sich besondere Vorteile durchsetzen können, etwa bei den Nutzungsquoten oder bei der Verteilung der Erhaltungskosten. Bei eigennützigen Akteuren stellen solche Asymmetrien die Stabilität der sozialen Norm, die die Nutzung der Ressource regelt, nicht in Frage, solange auch für die Benachteiligten normgemäßes Verhalten immer noch vorteilhafter ist als Normabweichung. Bewerten die Akteure die Interaktion allerdings auch unter Fairnessgesichtspunkten, dann kann - je nach Fairnessmaßstab - eine Asymmetrie der Verteilung als ein zusätzlicher negativer Nutzen zu Buche schlagen (Fehr/Schmidt, 1999), aufgrund dessen die reziproken Akteure trotz der materiellen Nachteile normabweichendes Verhalten vorziehen.

\section{d) Zentrales Recht und dezentrale soziale Normen}

Auch unter der Annahme von Fairnesspräferenzen ist die Interaktion von zentralem Recht und dezentralen sozialen Normen zunächst einmal eine Frage, ob sich die beiden Formen von Sanktionen gegenseitig stützen und stören. Soweit soziale Normen auf der Grundlage von bedingter Kooperation ein rechtliches Ressourcenregime stützen, ist es plausibel, dass soziale Normen einen größeren Beitrag leisten können, als dies unter eigennützigen Akteuren der Fall wäre. Umgekehrt ist die relative Last größer, die ein staatliches Nutzungsregime tragen muss, wenn es die Nutzung der Ressource ohne freiwillige Unterstützung durch die lokalen Akteure durchsetzen muss. Denn eine staatliche Intervention, die die Unterstützung vor Ort nicht genießt, muss diese freiwilligen Aufwendungen ausgleichen. Dabei wird nicht nur die geringere Bereitschaft zur Ressourcenschonung ins Gewicht fallen, sondern zu einem erheblichen Maß auch das geringere Ausmaß an dezentraler informaler Kontrolle, die von den Beteiligten vermutlich mit geringeren Aufwendungen geleistet werden kann als von staatlichen Behörden (vgl. Bandiera et al., 2005: 476).

\section{Heterogenität und Äquität}

\section{a) Heterogenität als Problem}

Wenn Akteure auf der Grundlage von Gegenseitigkeit kooperieren und jeder seine Nutzung einer gemeinschaftlichen Ressource auf einen fairen Anteil beschränkt, solange auch die anderen dies tun, dann setzt das implizit voraus, dass die Akteure die gleichen oder doch zumindest kompatible Auffassungen davon haben, welche Ressourcennutzung fair und welche unfair ist (Magen, 2006: 65). Kooperative Lösungen auf der Grundlage von Fairness brauchen also nicht nur technisches Wissen etwa über die optimale Entnahmemenge, sondern auch einen geteilten Maßstab dafür, welche Verteilung dieser Menge in dem relevanten sozialen Kontext als fair betrachtet wird. Eine derartige Übereinstimmung ist vergleichsweise einfach zu erzielen, wenn die Akteure weitgehend homogen sind, also wenn im hiesigen Beispiel die beteiligten Landwirte ungefähr 
gleich große Felder bewirtschaften und Pflanzen mit einem vergleichbaren Wasserbedarf anbauen. Dann liegt es nahe, dass fair bedeutet, jedem einen gleich großen Anteil am Wasser zuzubilligen. Die meisten Laborexperimente der Verhaltensökonomie modellieren eine solche Situation umfassender Gleichheit und können unter diesen Bedingungen in der Tat robust beobachten, wie bedingte Kooperation und die Sanktionierung von Trittbrettfahrern zu stabiler Kooperation führt.

Wie aber verhalten sich heterogene Akteure, zwischen denen also Unterschiede von solcher Art bestehen, dass auch andere Verteilungsregeln als die einer Gleichverteilung plausibel wären? Welche Folgen hat es etwa im hiesigen Beispiel für die freiwillige Kooperationsbereitschaft, wenn einer der Landwirte ein größeres Feld besitzt oder wenn er Pflanzen anbaut, die einen höheren Wasserbedarf haben, wenn er einen größeren Teil der Aufwendungen für die Wasserkanäle getragen hat, wenn er in der Vergangenheit ein besonderes Wasserrecht erworben hat oder wenn er einer sozial höher stehenden Kaste angehört? Wird es dann auch als fair angesehen, wenn er unter Bezug auf einen dieser Gründe eine relativ höhere Wasserzuteilung in Anspruch nimmt? Allgemeiner gefragt, können die Beteiligten eine auf Gegenseitigkeit gegründete freiwillige Kooperation auch unter den Bedingungen von Heterogenität aufrecht erhalten? Die wenigen ökonomischen Experimenten, die es zu dieser Frage gibt, mahnen insoweit zur Vorsicht, denn in ihnen ist die Kooperation unter heterogenen Akteuren erheblich fragiler (Cardenas, 2003; Cherry et al. 2005; Ledyard, 1995). Eine Vielzahl von Feldstudien über die Nutzung von Gemeingütern berichten ebenfalls, dass Gemeingutsregime bei größerer Heterogenität der Nutzer häufig - aber nicht immer - weniger erfolgreich sind. Bemerkenswert ist allerdings, dass die Übernutzung der Ressource keineswegs eine zwangsläufige Folge der Heterogenität ist, sondern durch gute Institutionen verhindert werden kann (Varughese/Ostrom, 2001; Ostrom, 2005b; Stern et al., 2002, 447; Bandiera et al., 2005; ähnlich die Experimente von Hackett et al., 1994). Die Frage ist deshalb, weshalb genau Heterogenität zu Problemen führt und welche Eigenschaften von Institutionen es sind, die bei ihrer Lösung helfen.

Von den verschiedenen relevanten Faktoren sollen hier allerdings nur Präferenzen für Fairness in den Blick genommen werden. Damit wird keineswegs in Abrede gestellt, dass manche Formen von Heterogenität zu problematischen materiellen Anreizen führen, etwa bei den bereits erwähnten Außenoptionen (Dayton-Johnson/Bardhan 2002; Varughese/Ostrom, 2001). Diese wichtige Dimension des Problems ist aber auch in Bezug auf Fairness interessant, weil Fairnesspräferenzen die Anreizprobleme möglicherweise vergrößern. Häufig werden Bemühungen zur Erhaltung von Gemeingütern durch Verteilungskämpfe zwischen den Betroffenen belastet (Acheson/Knight, 2000; Bardhan, 2005). In diesen Auseinandersetzungen sollten Ungleichgewichte in der Verhandlungsstärke zwischen rationalen Egoisten zwar zu ungleichen Verteilungen führen (Knight, 1992), aber nicht notwendig zu mehr Konflikt, weil sich eigennützige Verlierer mit dem maximal realisierbaren Anteil zufrieden geben. Hegt ein Teil der Betroffenen aber, wie die empirische Evidenz zeigt, eine Aversion gegenüber unfairen Verteilungen, können diese auch über das nutzenmaximierende Maß hinaus bereit sein, Ressourcen in Verteilungskonflikte zu investieren, um die Benachteiligung zu beseitigen (Ullmann-Margalit/Sunstein, 2002). Fairnesspräferenzen könnten also Verteilungskonflikte verschärfen. 


\section{b) Mehrdeutigkeit von Fairnesspräferenzen als eine Ursache}

Das lässt allerdings noch offen, nach welchem Maßstab die Akteure Fairness beurteilen. Verhaltensökonomische Fairnesstheorien unterstellen diesbezüglich in der Regel, die Teilnehmer hätten per se eine Aversion gegen ungleiche Verteilungen, würden also Fairness mit Gleichverteilung in eins setzen (vgl. Bolton/Ockenfels, 2000; Fehr/Schmidt, 1999). In Gemeingütern sollten dann ungleiche Nutzungsquoten immer zu Problemen führen. Gleichverteilung ist aber in Laborexperimenten nur dann der dominierende Fairnessmaßstab, wenn die Teilnehmer mit gleich guten Gründen Ansprüche auf die zu verteilende Masse erheben können. Hat aber etwa ein Teil der Teilnehmer aus Gründen, die als legitim empfunden werden, eine besondere Position, etwa weil sie in einem vorangegangenen Leistungstest besser abgeschnitten haben, wird auch eine Zuteilung von höheren Anteilen als fair akzeptiert (Hoffmann et al, 1994, 1998; Smith, 2003). In solchen Fällen legen die Akteure in der Regel proportionale Verteilungsregeln zugrunde (z.B. Hackett et al., 1994), wie sie die sozialpsychologische Äquitäts-Theorie (Equity-Theory) seit langem beschreibt (Röhl, 1992). Äquitätsregeln ermöglichen es also, Unterschiede zwischen den Akteuren bei den Verteilungsfragen zu berücksichtigen.

Die durch Heterogenität verursachten Probleme entstehen dann möglicherweise auf einer anderen Ebene, nämlich dadurch, dass in solchen Konstellationen kein eindeutiges Kriterium für Fairness existiert, welches den Akteuren eine koordinierte Bewertung des Verhaltens erlauben würde. Zwar liegen proportionalen Gerechtigkeitsurteilen nur eine geringe Zahl von Prinzipien zugrunde, nämlich Leistung, Bedürfnis, Berechtigung und Status. Die Notwendigkeit der Auswahl eines dieser Kriterien, seiner Operationalisierung und, im Fall mehrer einschlägiger Kriterien, ihre Konkurrenz, führen aber dazu, dass der Standard für Fairness jedenfalls nicht durch die allgemeine Struktur der Fairnesspräferenzen determiniert wird. Die Akteure wollen mit anderen Worten, dass zu fairen Bedingungen kooperiert wird, aber es ist auch für sie nicht immer deutlich, was fair ist. Der Maßstab für Fairness ist mithin keine individuell stabile Größe, sondern wechselt mit der jeweiligen Situation und ihrer sozialen Bedeutung (Liberman et al., 2004). Man könnte auch sagen, dass Fairness-Präferenzen unvollständig und deshalb auf Institutionen angewiesen sind. Fehlen solche, und ist der Inhalt von Fairness unklar oder ambivalent, dann legen die Akteure ihren normativen Erwartungen mit großer Robustheit denjenigen Fairnessmaßstab zugrunde, der für ihre eigenen Interessen am günstigsten ist (sog. „self-serving bias“; Konow, 2005). Eigen- und Fremdbewertung von Fairness treten dann auseinander und jeder nimmt die Nutzung des Gemeinguts durch die Anderen tendenziell als unfair wahr. Als Reaktion auf die wahrgenommene Unfairness wird dann die eigene Zurückhaltung aufgegeben und/oder das vermeintlich unfaire Verhalten der anderen sanktioniert. So kommt es zu den jedem Juristen vertrauten Bestrafungskaskaden, bei denen diejenigen, die Adressaten von Sanktionen geworden sind, mit Vergeltungsmaßnahmen antworten, weil sie ihr Verhalten als fair und die Sanktionen als Affront werten (vgl. Denant-Boemont, 2005). Diese Sicht auf die durch Heterogenität verursachten Probleme passt zu der Beobachtung, dass die Wirkung von Heterogenität wesentlich von der Qualität der Institutionen beeinflusst wird. Institutionen käme dann - neben anderem - die Funktion zu, die unter Heterogenität verschärfte Ambivalenz von Fairness zu vermindern, um 
die Angriffspunkte für eigennützige verzerrte Fairnesswahrnehmungen zu verringern und die gegenseitigen normativen Erwartungen zu koordinieren.

\section{c) Zentrales Recht und dezentrale Institutionen}

Institutionen könnten also unter Rückgriff auf Kriterien der Äquität (Leistung, Bedürfnis, Berechtigung, Status) klären, welche Relevanz faktische Unterschiede für die Beurteilung der Fairness von Verhalten in dem maßgeblichen sozialen Kontext zukommt. Damit ist noch nichts über die möglichen Quellen solcher Institutionen gesagt. Nicht selten werden sie endogen aus der Interaktion der Betroffenen hervorgehen, besonders wenn Gruppen über gewisse Zeiträume interagieren. Viele solcher informalen Institutionen können nicht auf bestimmte Entscheidungen zurückgeführt werden (Cooter, 1998). In vielen Fällen ist der Prozess der endogenen Produktion von Institutionen aber durchaus in variierenden Graden formalisiert. So werden nicht selten Nutzungsregeln für Gemeingüter auf Versammlungen oder in anderen Verfahren beschlossen. Erste Laborexperimenten stützen die Vermutung, dass solche formalen (aber nicht notwendig staatlichen bzw. zentralen) Institutionen sich auch in den Fairnesserwartungen der Beteiligten widerspiegeln (McAdams/Nadler, 2005; Gächter/Riedl, 2005; dazu Magen, 2006: 59 ff.). Große Bedeutung kommt dabei der Fairness des Verfahrens zu. Aus verschiedenen Gründen werden formale Regeln, die die Beteiligten selbst beschlossen haben, einfacher als legitim akzeptiert (vgl. Frey et al., 2004). Das impliziert eine größere Wirksamkeit lokaler Institutionen, schließt aber eine expressive Wirkung von staatlichen Regeln auf die Fairness-Präferenzen der Adressaten nicht aus (Magen, 2006).

\section{Adaptive Institutionen}

Nach hiesiger These ist es also eine Funktion von Institutionen, die Ambivalenz von Fairnesserwartungen zu verringern, damit Kooperation unter heterogenen Akteuren möglich wird. Davon ausgehend sollen abschließend noch einige Vermutungen zu der Frage formuliert werden, welche Eigenschaften von Institutionen dieser kooperationsfördernden Wirkung dienlich sind.

\section{a) Beschränkte Rationalität und einfache Institutionen}

Nicht immer treffen reale Akteure ihre Entscheidungen so, wie es Theorien vollständiger Rationalität beschreiben. Nicht nur müssen sie oft auf der Grundlage von unvollständigen Informationen und in knapper Zeit entscheiden. Sie verfügen auch nur über begrenzte kognitive Fähigkeiten und Kapazitäten (Gigerenzer/Selten, 2002). Jenseits der Frage, ob Menschen nur nach materiellen Vorteilen oder auch nach Fairness oder anderen Zielen streben, wäre es deshalb eine wenig realistische Vorstellung, die Akteure versuchten ihre Ziele im mathematischen Sinn optimal umzusetzen. Sieht man von professionellen Marktteilnehmern ab, werden sich die Akteure eher an einem realistischen Erwartungsniveau orientieren, dessen Erreichung als eine zufrieden 
stellende Verwirklichung ihrer Ziele erscheint, und dieses Erwartungsniveau im Laufe der Erfahrung anpassen (sog. Satisfizieren; vgl. Simon, 1957; Selten 2002). Welche Entscheidungen sie zu diesem Erwartungsniveau führen, bestimmen sie oft mit einfachen, aber bewährten („,adaptiven“) Entscheidungsregeln, die nur einen Teil der möglichen Informationen verarbeiten und keinen übermäßigen gedanklichen Aufwand erfordern (sog. Heuristiken; vgl. Gigerenzer/Todd, 1999). Die Begrenztheit dieser Rationalität liegt also vor allem darin, dass nicht mit komplizierten Kalkülen nach optimalen Entscheidungen gesucht wird. ${ }^{9}$ Ein solches Verhalten muss aber keineswegs unklug sein. Im Gegenteil können bewährte Entscheidungsregeln, wenn sie für das anstehende Problem richtig gewählt sind, zu praktisch guten Entscheidungen führen, in dem sie die für ein Problem relevanten Information aufgreifen und in einer Weise verarbeiten, die typischerweise sinnvoll ist. ${ }^{10}$

Wenn Akteure ihre Entscheidungen unter Rückgriff auf einfache Entscheidungsregeln und mit begrenztem kognitiven Aufwand und nach beschränkter Informationssuche treffen und nicht optimale, sondern nur zufriedenstellende Ergebnisse suchen, hat dies Bedeutung dafür, wie Institutionen Einfluss auf ihre Entscheidungen gewinnen können. Nicht nur sollten Institutionen mit der psychischen Struktur von Fairnesserwartungen kompatibel sein, wie sie sich insbesondere in den Grundsätzen der Äquitat spiegelt. Sie sollten auch kognitiv einfach zu rezipieren sein, damit sie in begrenzt rationale Entscheidungsprozesse Eingang finden können. Dabei ist auch zu berücksichtigen, dass die hier in Frage stehenden Institutionen sich an eine Vielzahl von Individuen richten, die in diversen Situationen miteinander interagieren und jeweils zu praktisch kompatiblen normativen Erwartungen finden müssen. Einfache Institutionen sind aber möglicherweise robuster, indem sie auch in unterschiedlichen Situationen zu praktikablen Ergebnissen führen (Kysar et al., 2006; Ostrom, 2005: 258 ff.).

Im Vergleich zum Prinzip der Gleichverteilung sind für Verteilungen auf der Grundlage von Äquität potentiell wesentlich mehr Gesichtspunkte in Betracht zu ziehen. Unter dem Aspekt begrenzter Rationalität wäre es aber wohl ein Irrweg, wenn Institutionen versuchten, möglichst viele der nach Äquitätsprinzipien potentiell relevanten Gesichtspunkte in möglichst differenzierter Weise zu berücksichtigen. Viel spricht vielmehr dafür, dass es für das Gelingen von Kooperation wichtig ist, äquitäre Fairnessprinzipien in einfacher und transparenter Weise umsetzen, damit ihre Bedeutung für die jeweiligen Interaktionen kognitiv transparent ist. Die zahlreichen Fallstudien, die zu realen Gemeingüter durchgeführt wurden, sprechen insoweit eine deutliche Sprache. Klare Regeln über die Nutzung der Ressource, so berichten sie, sind ein immer wieder genanntes Merkmal erfolgreicher Gemeingüterregime (Bandiera et al., 2005; Ostrom, 2005b; Dietz et al., 2003; Trawick, 2001). In ihnen sind Verteilungsfragen nach plastischen und in der Regel eindimensionalen Äquitätsregeln geregelt (Ostrom, 2005b; vgl. auch Ellickson, 1991: 72). Nicht untypisch ist es etwa, wenn knappes Wasser proportional zur Größe der Anbaufläche aufgeteilt wird (Trawick, 2002). Bemerkenswert an diesem Bedürfniskriterium ist, dass eine

$9 \quad$ Bei schlecht definierten oder zu komplizierten Problemen ist Optimieren zudem gar nicht möglich (Kysar et al., 2006).

10 Wenn nicht auch dieser Begriff besetzt wäre, würde man außerhalb von ökonomischen Diskursen deshalb statt von begrenzter vielleicht besser von praktischer Rationalität sprechen. 
Berücksichtigung vieler anderer Faktoren, die ebenfalls den individuellen Wasserbedarf beeinflussen können, unterbleibt. Typisch ist auch eine Verteilung von Infrastrukturkosten proportional dazu, wie viel Einheiten der Ressource entnommen wurden (Ostrom 2005b; Bandiera et al., 2005: 476; Bardhan, 2000; Dayton-Johnson, 2000). Jeweils wird durch plastische Regeln verhindert, dass Unklarheit über die Fairness oder Unfairness der jeweiligen Nutzung Anlass zu Streitigkeiten und Raum für eigennützige Situationsbewertungen geben und dadurch die freiwillige Kooperationsbereitschaft untergraben wird. Solange ein realistisches Erwartungsniveau an Fairness nicht unterschritten und grobe Ausbeutung verhindert wird, muss eine zusätzliches Mehr an Einzelfallgerechtigkeit im Aggregat der individuellen Erwartungen nicht immer zusätzliche Legitimität bedeuten, als vielmehr mehr Streit und weniger Kooperation.

\section{b) Transparenz der Normbefolgung}

Dass die Wirksamkeit von Normen damit zu tun hat, ob diese als legitim wahrgenommen werden, entspricht gängiger Vorstellung. So mag eine Norm umso eher befolgt werden, umso mehr sie mit den Werten der Adressaten übereinstimmt. Wenn informale Normen oder die Bereitschaft zur Befolgung formaler Normen aber auch auf einer generalisierten Gegenseitigkeit beruht, könnte der Zusammenhang auch in der umgekehrten Richtung statthaben, die Akzeptanz der Norm also umgekehrt vom Grad ihrer Durchsetzung abhängen (vgl. Kahan, 2005; Vandenbergh, 2005). Dass eine Norm befolgt wird, bedeutet dann nämlich, dass jeder seinen Beitrag zu dem von der Norm verfolgten Ziel leistet. Deshalb ist nicht nur der Inhalt einer Norm von Bedeutung, sondern auch, wie dieser sich operationalisieren lässt, weil die Operationalisierung wesentlichen Einfluss darauf hat, in welchen Maß die Normbefolgung für die anderen Beteiligten transparent wird (Ostrom, 2005b). Wie eine kluge Operationalisierung eines Äquitätsprinzips die Transparanz der Normbefolgung fördern kann, kann das Beispiel eines erfolgreichen Bewässerungssystems in den Hochanden verdeutlichen (Trawick, 2001, 2002). Dieses Bewässerungssystem besteht aus am Hang liegenden Terrassenfeldern, die mit einer niedrigen Umgrenzung von einheitlicher Höhe umschlossen sind. Die Wasserzuteilung erfolgt dann so, dass jedes Feld so lange bewässert wird, bis es bis zur Umgrenzung unter Wasser steht. So wird nicht nur auf einfache Weise sichergestellt, dass jedes Feld proportional die gleiche Menge Wasser erhält, sondern auch die Einhaltung dieser Norm für jedermann einsichtig gemacht.

\section{c) Anpassung an lokale Verhältnisse}

Institutionen zur Lösung von Gemeingutsdilemmata sind nach unserer These erfolgreicher, wenn sie transparente und robuste Fairnesskriterien umsetzen, deren Einhaltung im lokalen Kontext sichtbar und kontrollierbar ist, weil diese Umstände freiwillige Kooperation auf der Grundlage von Fairness und Gegenseitigkeit begünstigen. Funktionierende Institutionen müssen also Anforderungen auf verschiedenen Ebenen gerecht werden und zugleich einfach sein (vgl. Trawick, 2001, 12 ff.). Wenn sich die Einsichten der Forschungen über adaptive Entscheidungsregeln 
auch auf Institutionen übertragen lassen (dafür: Gigerenzer/Engel, im Erscheinen), dann sind damit keine unmöglichen Anforderungen formuliert. Voraussetzung ist freilich, dass die Entscheidungsregel an die Struktur des zu lösenden Problems angepasst ist. In dem Maße, wie die Probleme lokal variieren, wird es aber leichter sein, wirksame und zugleich einfache Institutionen auf dezentraler Ebene zu generieren. So können dezentrale Institutionen die lokal relevanten Verteilungsprobleme aufgreifen und in einer Weise lösen, die psychologisch plausibel und sozial praktikabel ist, aber mögliche Konfliktpunkte, die vor Ort keine Rolle spielen, unberücksichtigt lassen, und Probleme der Überwachung und Sanktionierung, die sich anderen Orts stellen mögen, außer Betracht lassen. Zumindest wird es oft notwendig sein, Operationalisierungen zu finden, die einfache Proportionalitätsregeln an die lokalen Verhältnisse anpassen.

Das läuft allerdings nicht auf die Empfehlung hinaus, Institutionen müssten ausschließlich jenseits des zentralen Rechts vor Ort gemacht werden. Es wird aber sinnvoll sein, die lokale Ebene mit der Befugnis auszustatten, formale Institutionen an die spezifischen Gegebenheiten des in Frage stehenden Gemeinguts anzupassen. So verfügen in der Praxis erfolgreiche Nutzungsregime zumeist über formale - aber nicht unbedingt staatliche - Mechanismen, um Regeln festzulegen und Konflikte zu lösen (Bandiera et al. 2005, 474; Ostrom, 2005: 258 ff.). Viel spricht dafür, dass jedenfalls bei der Erhaltung von Gemeingütern die Rechtsform - sei es der eines privaten Zusammenschlusses, eines autonomen öffentlichen Verband oder der einer mit den Betroffenen zusammenarbeitenden Zweigstelle einer staatlichen Behörde - zweitrangig ist gegenüber der sorgfältigen Anpassung des Regelwerks an die lokalen Verhältnisse (Dietz et al., 2003, Online Supplement). Es wäre deshalb irreführend, staatliche und dezentrale Institutionen nur als Gegensätze zu betrachten.

\section{Ausblick}

Für die Gestaltung praktikabler Institutionen interessiert weniger die grundsätzliche Frage, ob nun das Recht der Sitte folgt, oder die Sozialmoral dem Recht, als die konkreten Wirkmechanismen, die zwischen Recht und soziale Normen vermitteln. Nur ein kleiner Ausschnitt der vielen relevanten Mechanismen konnte hier angesprochen werden, nämlich Fairness und Gegenseitigkeit, und dies auch nur in Bezug auf die Bewältigung sozialer Dilemmata. Gegenseitigkeit zeigt sich in Laborexperimenten und Feldstudien als ein eigenständiges Motiv, aus dem soziale Normen zur Bewältigung sozialer Dilemmata entstehen. Deren Stabilität beruht wesentlich auf der Wahrnehmung, dass sich auch die Anderen kooperativ oder fair verhalten. Dafür muss klar sein, was fair ist, und unfaires Verhalten muss durch Sanktionen unterbunden werden.

Das impliziert zwei Ebenen, auf denen staatliches Recht und dezentrale soziale Normen interagieren, nämlich auf der Ebene der Sanktionen und auf der Ebene der Fairnessstandards. Das Recht kann zum einen informale Sanktionen untergraben oder unterstützen oder die betroffenen Akteure subsidiär mit rechtlich vermittelter Sanktionsgewalt ausstatten. Zum anderen bedarf freiwillige Kooperation auf der Grundlage von Gegenseitigkeit eines geteilten Maßstabs für Fairness. Besteht zwischen den Akteuren Heterogenität, ist es deshalb erforderlich, dass die 
Akteure zu einem gemeinsamen Verständnis darüber gelangen, wie die Unterschiede in den Fairnesserwartungen berücksichtigt werden sollen. Dafür greifen die Akteure auf Prinzipien der Äquität zurück, müssen sich aber darüber abstimmen, welche der Prinzipien wie zur Anwendung kommen sollen, weil ansonsten eigennützig verzerrte Fairnesserwartungen eine Kooperation erschweren. Die Aufgabe, die Akteure über die geteilten Fairnessmaßstäbe zu orientieren, kann formalen oder informalen Institutionen zufallen. Vermutlich wird kooperatives Verhalten in sozialen Dilemmata gefördert, wenn institutionalisierte Verteilungsregeln Prinzipien der Äquität in einfache, an die lokalen Gegebenheiten angepasste, transparente und robuste Entscheidungsregeln umsetzen. 


\section{Literatur}

Acheson, James M./Knight, Jack, 2000: Distribution Fights, Coordination Games, and Lobster Management, in: Comparative Studies in Society and History 42, S. 209

Arce, Daniel G./Sandler, Todd, 2005: The Dilemma of the Prisoners’ Dilemma, in: Kyklos 58, S. 3

Aretz, Hans-Jürgen, 2005: Die Relevanz von Wertverpflichtungen bei der Bereitstellung öffentlicher Güter, in: Zeitschrift für Soziologie 34, S. 326

Axelrod, Robert K., 1984: The Evolution of Cooperation, New York

Baird, Douglas/Gertner, Robert/Picker, Randal, 1998: Game Theory and the Law, 3. Aufl., Cambridge, Mass.

Bandiera, Oriana/Barankay, Iwan/Rasul, Imran, 2005: Cooperation in Collective Action, in: Economics of Transition 13, S. 473

Bardhan, Pranab, 2000: Irrigation and Cooperation: An Empirical Analysis of 48 Irrigation Communities in South India, in: Economic Development and Cultural Change 48, S. 847

Bardhan, Pranab, 2005: Institutions Matter, But Which Ones?, in: Economics of Transition 13, S. 499

Beckenkamp, Martin, 2002: A Game Theoretic Taxonomy of Social Dilemmas, Max-PlanckInstitut zur Erforschung von Gemeinschaftsgütern, Preprint 2002/11, Bonn

Beckert, Jens, 1997: Grenzen des Marktes, Frankfurt am Main

Beckert, Jens, 2003: Economic Sociology and Embeddedness, in: Journal of Economic Issues 3, S. 769

Beckert, Jens, 2005: The Moral Embeddedness of Markets, Max-Planck-Institut für Gesellschaftsforschung, Discussion Paper 05/6, Köln

Bolton, Gary E./Ockenfels, Axel, 2000: ERC: A Theory of Equity, Reciprocity, and Cooperation, in: American Economic Review 90, S. 166

Bowles, Samuel, 2004: Microeconomics, Princeton, NJ

Camerer, Colin F., 2003: Behavioral Game Theory, Princeton, NJ

Cardenas, Juan-Camilo, 2003: Real Wealth and Experimental Cooperation, in: Journal of Development Economics 70, S. 263 
Cherry, Todd L./Kroll, Stephan/Shogren, Jason F., 2005: The Impact of Endowment Heterogeneity and Origin on Public Good Contributions: Evidence From the Lab, in: Journal of Economic Behavior and Organization 57, S. 357

Coleman, James S., 1990: The Emergence of Norms, in: Michael Hechter/Karl-Dieter Opp/Reinhard Wippler, Social Institutions, New York, S. 35

Cooter, Robert D., 1998: Expressive Law and Economics, Journal of Legal Studies 27, S. 585

Cooter, Robert D., 2000: Three Effects of Social Norms on Law: Expression, Deterrence, and Internalization, in: Oregon Law Review 79, S. 1

Dawes, Robyn N., 1980: Social Dilemmas, in: Annual Review of Psychology 31, S. 169

Dayton-Johnson, Jeff, 2000: Determinants of Collective Action on the Local Commons: a Model with Evidence from Mexico, in: Journal of Development Economics 62, S. 181

Dayton-Johnson, Jeff/Bardhan, Pranab, 2002: Inequality and Conservation on the Local Commons, in: The Economic Journal 112, S. 577

Denant-Boemont, Laurent/Masclet, David/Noussair/Charles, 2005: Punishment, Counterpunishment and Sanction Enforcement in a Social Dilemma Experiment, Working Paper, Emory University, Atlanta

Dietz, Thomas/Dolsak, Nives/Ostrom, Elinor/Stern, Paul, 2002: The Drama of the Commons, in: Elinor Ostrom et al. (Hrsg.), The Drama of the Commons, Washington, DC, S. 3

Dietz, Thomas/Ostrom, Elinor/Stern, Paul, 2003: The Struggle to Govern the Commons, in: Science 302, S. 1907

Ellickson, Robert C., 1991: Order Without Law, Cambridge, Mass.

Engel, Christoph/Gigerenzer, Gerd (Hrsg.), im Erscheinen: Heuristics and the Law. Cambridge, Mass. 2005

Fehr, Ernst /Fischbacher, Urs, 2003: The Nature of Human Altruism, in: Nature 425, S. 785

Fehr, Ernst/Fischbacher, Urs, 2004: Third-Party Punishments and Social Norms, in: Evolution and Human Behavior 25, S. 63

Fehr, Ernst/Gächter, Simon, 2000a: Cooperation and Punishment in Public Goods Experiments, in: American Economic Review 90, S. 980

Fehr, Ernst /Gächter, Simon, 2000b: Fairness and Retaliation: The Economics of Reciprocity, in: Journal of Economic Perspectives 14, S. 159 
Fehr, Erns/Schmidt, Klaus, 1999: A Theory of Fairness, Competition and Cooperation, in: Quarterly Journal of Economics 114 (1999), S. 817

Fischbacher, Urs/Gächter, Simon/Fehr, Ernst, 2001: Are People Conditionally Cooperative? Evidence from a Public Goods Experiment, in: Economics Letters 71, S. 397

Frey, Bruno/Benz, Matthias/Stutzer, Alois, 2004: Introducing Procedural Utility, in: Journal of Institutional and Theoretical Economics 160, S. 377

Fritsch, Michael/Wein, Thomas/Ewers, Hans-Jürgen, 2005: Marktversagen und Wirtschaftspolitik, 6. Aufl., München

Gächter, Simon/Riedl, Arno, 2005: Moral Property Rights in Bargaining with Infeasible Claims, Management Science 51, S. 249

Gigerenzer, Gerd/Selten, Reinhard (Hrsg.), 2002: Bounded Rationality, Cambridge, Mass.

Gigerenzer, Gerd/Todd, Peter M., 1999: Simple Heuristics that Make us Smart, Oxford

Hackett, Steven/Schlager, Edella/Walker, James, 1994: The Role of Communication in Resolving Commons Dilemmas: Experimental Evidence with Heterogeneous Appropriators, in: Journal of Environmental Economics and Management 27, S. 99

Hardin, Garrett, 1968: The Tragedy of the Commons, in: Science 162, S. 1243

Hardin, Russell, 1982: Collective Action, Baltimore

Hardin, Russell, 2000: Law and Social Norms in the Large, Virginia Law Review 86, S. 1821

Heckathorn, Douglas D., 1989: Collective Action and the Second-Order Free-Rider Problem, in: Rationality and Society 1, S. 78

Heckathorn, Douglas D., 1996: The Dynamics and Dilemmas of Collective Action, in: American Sociological Review 61, S. 250

Hechter Michael, 1990: The Emergence of Cooperative Social Institutions, in: ders./Karl-Dieter Opp/Reinhard Wippler, Social Institutions, New York, S. 13

Hechter, Michael/Opp, Karl-Dieter, 2001: Introduction, in: dies. (Hrsg.) Social Norms, New York, S. xi

Hoffman, Elizabeth/McCabe, Kevin/Shachat, Keith/Smith, Vernon L., 1994: Preferences, Property Rights, and Anonymity in Bargaining Games, in: Games and Economic Behavior 7, S. 346

Hoffman, Elizabeth/McCabe, Kevin/ Smith, Vernon L., 1998: Behavioral Foundations of Reciprocity, in: Economic Inquiry 36, S. 335 
Kahan, Dan, 2005: The Logic of Reciprocity: Trust, Collective Action, and Law, in: Herbert Gintis/Samuel Bowles/Robert Boyd/Ernst Fehr (Hrsg.), Moral Sentiments and Material Interests, Cambridge, Mass., S. 339

Kirchgässner, Gebhard, 2000: Homo Oeconomicus, 2. Aufl., Tübingen

Knight, Jack, 1992: Institutions and Social Conflict, Cambridge, UK

Kollock, Peter, 1998: Social Dilemmas: The Anatomy of Cooperation, in: Annual Review of Sociology 24, S. 183

Konow, James, 2005: Blind Spots: The Effects of Information and Stakes on Fairness Biases and Dispersion, Social Justice Research 18, 349

Kreps, David, 1990: Game Theory and Economic Modelling, Oxford

Kurzban, Robert/Houser, Daniel, 2001: Individual Differences in Cooperation in a Circular Public Goods Game, in: European Journal of Personality 15, S. S37

Kysar, Douglas/Ayton, Peter/Frank, Robert H./Frey, Bruno S./Gigerenzer, Gerd/Glimcher, Paul W./Korobkin, Russell/Langevoort, Donald C./Magen, Stefan, 2006: Are Heuristics a Problem or a Solution, in: Christoph Engel/Gerd Gigerenzer (Hrsg.), Heuristics and the Law. Cambridge, Mass, im Erscheinen

Ledyard, John O., 1995: Public Goods: A Survey of Experimental Research, in: John H. Kagen/ Alvin E. Roth (Hrsg.), Handbook of Experimental Economics, Princeton 1995, S. 111

Liberman, Varda/Samuels, Steven M./Ross, Lee, 2004: The Name of the Game: Predictive Power of Reputations versus Situational Labels in Determining Prisoner's Dilemma Game Moves, in: Personality and Social Psychology Bulletin 30, S. 1175

Lübbe-Wolff, Gertrude, 1999: Recht und Moral im Umweltschutz, Baden-Baden

Macy, Michael W./Flache, Andreas, 2002: Learning Dynamics in Social Dilemmas, in: Proceedings of the National Academy of Science 99, S. 7229

Magen, Stefan, 2006: Fairness, Eigennutz und die Rolle des Rechts, in: Christoph Engel et al. (Hrsg.), Recht und Verhalten, Tübingen (im Erscheinen); zitiert nach: Preprint des MaxPlanck-Instituts zur Erforschung von Gemeinschaftsgütern Nr. 2005/22, Bonn 2005

Mantzavinos, Chrysostomos, 2001: Individuals, Institutions, and Markets, Cambridge, UK

McAdams, Richard H./Nadler, Janice, 2005: Testing the Focal Point Theory of Legal Compliance, in: Journal of Empirical Legal Studies 2 (2005), S. 87

Olson, Mancur, 1998 : Die Logik kollektiven Handelns, 4. Aufl., Tübingen 
Opp, Karl-Dieter, 2001: Social Networks and the Emergence of Protest Norms, in: Michael Hechter/ders., Social Norms, New York, S. 234

Ostrom, Elinor, 1997: Governing the Commons, Cambridge, UK

Ostrom, Elinor, 2000: Reformulating the Commons, in: Swiss Political Science Review 6, S. 29

Ostrom, Elinor, 2003: Toward a Behavioral Theory Linking Trust, Reciprocity, and Reputation, in: dies./James Walker (Hrsg.), Trust and Reciprocity, New York, S. 19

Ostrom, Elinor, 2005a: Policies That Crowd out Reciprocity and Collective Action, in: Herbert Gintis/Samuel Bowles/Robert Boyd/Ernst Fehr (Hrsg.), Moral Sentiments and Material Interests, Cambridge, Mass., S. 253

Ostrom, Elinor, 2005b: Understanding Institutional Diversity, Princeton

Ostrom, Elinor/Gardner, Roy/Walker, James, 1994: Rules, Games, and Common-Pool Resources, Ann-Arbor

Ostrom, Elinor/Burger, Joanna/Field, Christopher B./Norgaard, Richard B./Policansky, David, 1999: Revisiting the Commons. Science 284, S. 278

Ostrom, Elinor/Dietz, Thomas/Dolsak, Nives/Stern, Paul C./Stonich, Susan/Weber, Elke (Hrsg.), 2002: The Drama of the Commons, Washington, DC

Rasmusen, Eric, 1994: Games and Information, 2. Aufl., Cambridge, Mass.

Rege, Mari, 2004: Social Norms and Private Provision of Public Goods, in: Journal of Public Economic Theory 6, S. 65

Röhl, Klaus F., 1992: Die Gerechtigkeitstheorie des Aristoteles aus der Sicht sozialpsychologischer Gerechtigkeitsforschung, Baden-Baden

Savigny, Friedrich Carl von, 1997 (1814): Vom Beruf unserer Zeit für Gesetzgebung und Rechtswissenschaft, Goldbach

Selten, Reinhard, 2002: What is Bounded Rationality?, in: Gerd Gigerenzer/Reinhard Selten (Hrsg.), Bounded Rationality, Cambridge, Mass., S. 13

Simon, Herbert A., 1957: Models of Man, New York

Smith, Vernon L., 2003: Constructivist and Ecological Rationality in Economics, in: American Economic Review 93, S. 465

Sumner, William G., 1940: Folkways, 3. Aufl., New York 
Stern, Paul/Dietz, Thomas/Dolsak, Nives/Ostrom, Elinor/Stonich, Susan, 2002: Knowledge and Questions after 15 Years of Research, in: Elinor Ostrom et al. (Hrsg.), The Drama of the Commons, Washington, DC, S. 445

Strahilevitz, Lior Jacob, 2003: Social Norms from Close-Knit Groups to Loose-Knit Groups, in: University of Chicago Law Review 70, S. 359

Tamanaha, Brian Z., 2001: A General Jurisprudence of Law and Society, Oxford

Trawick, Paul, 2001: Successfully Governing the Commons, in: Human Ecology 29, 1

Trawick, Paul, 2002: Comedy and Tragedy in the Andean Commons, in: Journal of Political Ecology 9, S. 35

Traxler, Christian, 2006: Tax Evasion, Social Norms and Conditional Cooperation, Diss. oec., Universität München (in Vorbereitung)

Ullmann-Margalit, Edna/Sunstein, Cass R., 2001: Inequality and Indignation, in: Philosophy and Public Affairs 30, S. 337

Vandenbergh, Michael P., 2005: Order Without Social Norms, in: Northwester University Law Review 99, 1101

Varughese, George/Ostrom, Elinor; 2001: The Contested Role of Heterogeneity in Collective Action, in: World Development 29, 747

Voss, Thomas, 2001: Game-Theoretical Perspectives on the Emergence of Norms, in: Michael Hechter/Karl-Dieter Opp (Hrsg.), Social Norms, New York, S. 105

Weimann, Joachim, 1995: Umweltökonomik, 3. Aufl., Berlin 